Mathematical Research Letters 1, 75-86 (1994)

\title{
MOMENT ESTIMATES DERIVED FROM POINCARÉ AND LOGARITHMIC SOBOLEV INEQUALITIES
}

\author{
S. Aida And D. Stroock
}

\section{Introduction}

In the analysis of probability measures in an infinite dimensional context, it is usually necessary to begin by getting some sort of estimate on the extent to which the measure under consideration differs from one which admits coordinates which are mutually independent. That is, one would like to know how mixing the measure is. For example, in the analysis of Gibbs states, such considerations sometimes allow one to determine whether one is in the single phase region (e.g. see [4] and [6]).

When the space on which the probability measure $\mu$ is defined possesses a natural gradient operation $\nabla$, a test for mixing properties is to examine what, if any, a priori estimates $\mu$ satisfies relative to $\nabla$. For example, one can ask whether $\mu$ satisfies a Poincaré inequality

$$
\left\|f-\mathbb{E}^{\mu}[f]\right\|_{L^{2}(\mu)}^{2} \leq P\|\nabla f\|_{L^{2}(\mu)}^{2} .
$$

Actually, (0.1) is the least one can ask of the relationship between $\mu$ to $\nabla$ : it simply measures the gap in the spectrum of the operator on $L^{2}(\mu)$ for which $\|\nabla f\|_{L^{2}(\mu)}^{2}$ is the quadratic form. A much stronger relationship between $\mu$ and $\nabla$ is expressed by the logarithmic Sobolev inequality

$$
\int f^{2} \log \frac{f^{2}}{\|f\|_{L^{2}(\mu)}^{2}} d \mu \leq S\|\nabla f\|_{L^{2}(\mu)}^{2} .
$$

Indeed, not only does (0.2) imply $(0.1)$ with $2 P \leq S$, it says that control on the $L^{2}(\mu)$-norm of $|\nabla f|$ gives one control on slighlty more than the $L^{2}(\mu)$-norm of $f$. Thus, $(0.2)$ is doing double duty: it is simultaneously

Received November 2, 1993. 
measuring a spectral gap and providing a coercivity statement. The coercivity aspect of (0.2) is segregated from the spectral gap aspect in the defective logarithmic Sobolev inequality

$$
\int f^{2} \log \frac{f^{2}}{\|f\|_{L^{2}(\mu)}^{2}} d \mu \leq S\|\nabla f\|_{L^{2}(\mu)}^{2}+D\|f\|_{L^{2}(\mu)}^{2} .
$$

The purpose of this note is to show that each of these a priori estimates allows one to obtain bounds on $f$ in terms of bounds on $|\nabla f|$. At least in cases when $\nabla$ is a true gradient, in the sense that it is local and therefore satisies the chain rule, essentially all of our results have been anticipated elsewhere. Thus, for the most part, the only new thing here is the methodology, which we believe is both simpler and more flexible than that used previously. Section 3 contains an example of the of the kind of applications which this increased flexibility affords.

\section{The setting}

In this article we will be dealing with the following abstract setting. $(E, \mathcal{B}, \mu)$ is a probability space and $(t, x) \longmapsto P(t, x, \cdot)$ is a Markov transition probability function on $(E, \mathcal{B})$ (i.e. $P(t, x, \cdot)$ is a probability measure on $(E, \mathcal{B})$ for each $(t, x) \in(0, \infty) \times E,(t, x) \in(0, \infty) \times E \longmapsto P(t, x, \Gamma)$ is $\mathcal{B}_{(0, \infty)} \times \mathcal{B}$-measurable for each $\Gamma \in \mathcal{B}$, and the Chapman-Kolmogorov equation holds) such that, for each $t \in(0, \infty)$, the probability measure $M_{t}$ on $\left(E^{2}, \mathcal{B}^{2}\right)$ given by

$$
M_{t}(d x \times d y)=P(t, x, d y) \mu(d x)
$$

is symmetric and, for each $f \in B(E)$ (the space of bounded, $\mathcal{B}$-measurable, $\mathbb{R}$-valued functions on $E$ )

$$
P_{t} f \equiv \int_{E} f(y) P(t, \cdot, d y) \longrightarrow f \text { in } L^{2}(\mu) \text { as } t \searrow 0
$$

As is well-known, this means that each $P_{t}$ admits a unique extension as a self-adjoint contraction $\bar{P}_{t}$ on $L^{2}(\mu)$, that $\left\{\bar{P}_{t}: t>0\right\}$ is strongly continuous, and, therefore, by Stone's Theorem, that

$$
\bar{P}_{t}=\int_{[0, \infty)} e^{-\lambda t} d E_{\lambda}
$$


where $\left\{E_{\lambda}: \lambda \in[0, \infty)\right\}$ is a resolution of the identity in $L^{2}(\mu)$ by orthogonal projections. In particular, for each $f \in L^{2}(\mu)$,

$$
\begin{aligned}
\frac{1}{2 t} \int_{E^{2}}(f(y)-f(x))^{2} & M_{t}(d x \times d y) \\
= & \left(f, f-\bar{P}_{t} f\right)_{L^{2}(\mu)} \\
& \nearrow \mathcal{E}(f, f) \equiv \int_{(0, \infty)} \lambda d\left(E_{\lambda} f, f\right)_{L^{2}(\mu)}
\end{aligned}
$$

as $t \searrow 0$. The quadratic form $\mathcal{E}$ is called the Dirichlet form, and we will use $D(\mathcal{E})$ to denote its domain: the space of $f \in L^{2}(\mu)$ for which $\mathcal{E}(f, f)<\infty$. Next, we define $\mathcal{E}(f, g)$, for $f, g \in D(\mathcal{E})$, by polarization:

$$
\mathcal{E}(f, g) \equiv \frac{1}{4}[\mathcal{E}(f+g, f+g)-\mathcal{E}(f-g, f-g)]=\int_{(0, \infty)} \lambda d\left(E_{\lambda} f, g\right)_{L^{2}(\mu)}
$$

Clearly, for all $f, g \in D(\mathcal{E})$ :

$$
\begin{aligned}
|\mathcal{E}(f, g)| & \leq \sqrt{\mathcal{E}(f, f)} \sqrt{\mathcal{E}(g, g)} \text { and } \\
\sqrt{\mathcal{E}(f+g, f+g)} & \leq \sqrt{\mathcal{E}(f, f)}+\sqrt{\mathcal{E}(g, g)} .
\end{aligned}
$$

In addition, one has that

$$
\mathcal{E}(f, g)=\lim _{t \searrow 0} \frac{1}{2 t} \int_{E^{2}}(f(y)-f(x))(g(y)-g(x)) M_{t}(d x \times d y) .
$$

Finally, it is an easy matter to check that $D(\mathcal{E})$ becomes a Hilbert space with the Hilbert norm

$$
\|f\|_{D(\mathcal{E})}=\sqrt{\|f\|_{L^{2}(\mu)}^{2}+\mathcal{E}(f, f)} .
$$

Notice that, as a consequence of the second expression in (1.4), one knows that for any $f \in D(\mathcal{E})$ and any uniformly Lipschitz continuous $\varphi$ on $\mathbb{R}, \varphi \circ f \in D(\mathcal{E})$ and

$$
\mathcal{E}(\varphi \circ f, \varphi \circ f) \leq L^{2} \mathcal{E}(f, f),
$$

where $L$ is the Lipshitz constant for $\varphi$. Thus, the space $\mathcal{F}=D(\mathcal{E}) \cap B(E)$ is both a lattice and an algebra. Moreover, if, for $n \in \mathbb{Z}^{+}$,

$$
\psi_{n}(t)= \begin{cases}-n & \text { when } t \leq-n \\ t & \text { when }-n<t<n \\ n & \text { when } t \geq n,\end{cases}
$$


then for each $f \in D(\mathcal{E})$ the function $f_{n}=\psi_{n} \circ f \in \mathcal{F}$. In fact, on the one hand, since $f_{n} \longrightarrow f$ in $L^{2}(\mu), \mathcal{E}(f, f) \leq \underline{\lim }_{n \rightarrow \infty} \mathcal{E}\left(f_{n}, f_{n}\right)$, while on the other hand, by $(1.8), \mathcal{E}\left(f_{n}, f_{n}\right) \leq \mathcal{E}\left(f_{n}, f\right) \leq \mathcal{E}(f, f)$. Hence, it is easy to check that

$$
\left\|\psi_{n} \circ f-f\right\|_{D(\mathcal{E})} \longrightarrow 0 \quad \text { as } n \rightarrow \infty .
$$

In particular, $\mathcal{F}$ is dense in $D(\mathcal{E})$.

Given $f \in B(E)$, define

$$
\left[\Gamma_{t}(f, f)\right](x)=\frac{1}{2 t} \int_{E}(f(y)-f(x))^{2} P(t, x, d y),
$$

$(t, x) \in(0, \infty) \times E$, note that

$$
g \Gamma_{t}(f, f)=\Gamma_{t}(g f, f)+\frac{1}{2} \Gamma_{t}\left(g, f^{2}\right),
$$

and conclude that

$$
\lim _{t \searrow 0} \int_{E} g \Gamma_{t}(f, f) \mu=\Lambda_{f}(g) \equiv \mathcal{E}(g f, f)-\frac{1}{2} \mathcal{E}\left(g, f^{2}\right), \quad f, g \in \mathcal{F} .
$$

In particular, for each $f \in \mathcal{F}, \Lambda_{f}$ is a non-negative, bounded linear functional on $\mathcal{F}$. Given $f \in \mathcal{F}$ and $p \in[2, \infty]$, define $\|f\|_{p} \geq 0$ so that

$$
\begin{gathered}
\|f\|_{p}^{2}=\sup \left\{\Lambda_{f}(g): g \in \mathcal{F} \text { and }\|g\|_{L^{q}(\mu)}=1\right\}, \\
\text { where } q=\left(\frac{p}{2}\right)^{\prime}=\frac{p}{p-2},
\end{gathered}
$$

and take $\mathfrak{D}_{p}=\left\{f \in \mathcal{F}:\|f\|_{p}<\infty\right\}$. Finally, define $\overline{\mathfrak{D}}_{p}$ to be the set of measurable $f$ on $E$ for which there exists a sequence $\left\{f_{n}\right\}_{1}^{\infty} \subseteq \mathfrak{D}_{p}$ with the properties that $f_{n} \longrightarrow f$ in $\mu$-measure and $\sup _{n}\left\|f_{n}\right\|_{p}<\infty$. Given $f \in \overline{\mathfrak{D}}_{p},\|f\|_{p}$ is defined so that $A>\|f\|_{p}$ if and only if there exists such a $\left\{f_{n}\right\}_{1}^{\infty}$ with $A>\underline{\lim }_{n \rightarrow \infty}\left\|f_{n}\right\|_{p}$. Note that (cf. (1.9)),

$$
\Gamma_{t}\left(\psi_{n} \circ f, \psi_{n} \circ f\right) \leq \Gamma_{t}(f, f), \quad n \in \mathbb{Z}^{+}, t \in(0, \infty), \text { and } f \in B(E),
$$

and conclude that

$$
\sup _{n}\left\|\psi_{n} \circ f\right\|_{p} \leq\|f\|_{p} \quad \text { for any } p \in[2, \infty] \text { and } f \in \overline{\mathfrak{D}}_{p} .
$$

The following estimate will play an important role in our analysis. Namely, $\varphi$ is a continuous, convex function on $\mathbb{R}$ and $f \in \mathfrak{D}_{p}$, then

$$
\begin{aligned}
& \mathcal{E}(\varphi \circ f, \varphi \circ f) \\
& \quad \leq 2\|f\|_{p}^{2} \begin{cases}\left\|\varphi^{\prime} \circ f\right\|_{2\left(\frac{p}{2}\right)^{\prime}}^{2} & \text { if } \varphi \text { is monotone } \\
\left\|\varphi^{\prime} \circ \mid f\right\|_{2\left(\frac{p}{2}\right)^{\prime}}^{2} & \text { if } \varphi \text { achieves its minimum at } 0 .\end{cases}
\end{aligned}
$$


To see (1.15), first note that it suffices to treat the case when $\varphi \in C^{2}(\mathbb{R})$ and therefore that $\varphi \circ f \in \mathcal{F}$. Next, assume, in addition, that $\varphi$ is nondecreasing. Then

$$
\begin{aligned}
\int_{E^{2}}(\varphi \circ f(y) & -\varphi \circ f(x))^{2} M_{t}(d x \times d y) \\
& =2 \int_{f(y)<f(x)}(\varphi \circ f(x)-\varphi \circ f(y))^{2} M_{t}(d x \times d y) \\
& \leq 4 t \int_{E}\left(\varphi^{\prime} \circ f\right)^{2} \Gamma_{t}(f, f) d \mu
\end{aligned}
$$

and get (1.15) by dividing through by $2 t$ and taking $t \searrow 0$. The other cases are handled in the same way, only with $\{f(y)<f(x)\}$ replaced by $\{f(x)<f(y)\}$ when $\varphi$ is non-increasing and by $\{|f(y)|<|f(x)|\}$ when $\varphi$ achieves its minimum at 0 .

Remark 1.17. In the case when the Dirichlet form is local, one has that, for any $\varphi \in C^{1}(\mathbb{R})$ and $f \in \mathcal{F}$,

$$
\mathcal{E}(\varphi \circ f, \varphi \circ f)=\Lambda_{f}\left(\left(\varphi^{\prime} \circ f\right)^{2}\right)
$$

and therefore that (1.6) can be replaced by

$$
\mathcal{E}(\varphi \circ f, \varphi \circ f) \leq\left\|\varphi^{\prime} \circ f\right\|_{L^{p}(\mu)}^{2}\|f\|_{2 \frac{p^{\prime}}{2}}
$$

\section{The Poincaré inequality}

We will say that $\mu$ satisies a Poincaré inequality relative to $\mathcal{E}$ if

$$
\begin{gathered}
P \equiv \sup \left\{\operatorname{var}(f): f \in L^{2}(\mu) \text { and } \mathcal{E}(f, f) \leq 1\right\}<\infty, \\
\text { where } \operatorname{var}(f) \equiv\|f-\langle f\rangle\|_{L^{2}(\mu)}^{2}
\end{gathered}
$$

and we have introduced the notation $\langle f\rangle$ to denote $\int f d \mu$. In this section we will investigate what can be said on the basis of a Poincaré inequality, and for this purpose we will need the following simple observation.

Lemma 2.2. Let $f \in L^{2}(\mu)$, and, for some $R \in(0, \infty)$ and $\theta \in(0$,$] ,$ assume that $\mu(X \leq R) \wedge \mu(X \geq-R) \geq \theta$. Then

$$
|\langle f\rangle| \leq R+\sqrt{\frac{\operatorname{var}(f)}{\theta}} \quad \text { and } \quad\|f\|_{L^{2}(\mu)} \leq R+2 \sqrt{\frac{\operatorname{var}(f)}{\theta}} \text {. }
$$


In particular, if $\left\{f_{n}\right\}_{1} \subseteq L^{2}(\mu)$ with $\sup _{n} \operatorname{var}\left(f_{n}\right)<\infty$ and if $f_{n} \longrightarrow f$ in $\mu$-measure, then $\sup _{n}\left\|f_{n}\right\|_{L^{2}(\mu)}<\infty$ and so $f \in L^{2}(\mu)$.

Proof. Clearly it suffices to prove the first inequality in (2.3), and, since both the hypotheses and conclusions are invariant under replacement of $f$ by $-f$, we will assume that $\langle f\rangle \geq 0$. If $\langle f\rangle \leq R$, then there is nothing to do. On the other hand, if $\langle f\rangle>R$, then

$$
(\langle f\rangle-R)^{2} \mu(f \leq R) \leq \int_{f \leq R}(\langle f\rangle-f)^{2} d \mu \leq \operatorname{var}(f)
$$

Theorem 2.4. Assume (2.1). Then $\overline{\mathfrak{D}}_{2}=D(\mathcal{E})$ and $\|f\|_{2}^{2}=\mathcal{E}(f, f)$.

Proof. Let $f \in \overline{\mathfrak{D}}_{2}$ be given, and choose $\left\{f_{\ell}\right\}_{1}^{\infty} \subseteq \mathfrak{D}_{2}$ so that $f_{\ell} \longrightarrow f$ in $\mu$-measure and $A=\sup _{\ell}\left\|f_{\ell}\right\|_{2}<\infty$. Next, define $\psi_{n}$ as in (1.9), and set $f_{\ell, n}=\psi_{n} \circ f_{\ell}$. Obviously, for each $n \in \mathbb{Z}^{+}, f_{\ell, n} \longrightarrow \psi_{n} \circ f$ in $\mu$-measure and therefore in $L^{2}(\mu)$ as $\ell \rightarrow \infty$, and, by (1.8), $\sup _{\ell, n} \mathcal{E}\left(f_{\ell, n}, f_{\ell, n}\right) \leq A^{2}$. Hence, by $(2.1), \sup _{\ell, n} \operatorname{var}\left(f_{\ell, n}\right) \leq P A^{2}$, and so, by Lemma 2.2, we now know that $\sup _{\ell, n}\left\|f_{\ell, n}\right\|_{L^{2}(\mu)}<\infty$. But this, combined with

$$
\lim _{\ell \rightarrow \infty}\left\|f_{\ell, n}-\psi_{n} \circ f\right\|_{L^{2}(\mu)} \longrightarrow 0 \quad \text { for each } n \in \mathbb{Z}^{+},
$$

means first that $\sup _{n}\left\|\psi_{n} \circ f\right\|_{D(\mathcal{E})}<\infty$ and then, because $\| \psi_{n} \circ f-$ $f \|_{L^{2}(\mu)} \longrightarrow 0$, that $\mathcal{E}(f, f) \leq \underline{\lim }_{n \rightarrow \infty} \mathcal{E}\left(\psi_{n} \circ f, \psi_{n} \circ f\right)<\infty$. Finally, since $\|f\|_{2}^{2}=\mathcal{E}(f, f)$ for $f \in \mathcal{F}$, it is easy to check that the same equality holds for $f \in D(\mathcal{E})$.

Theorem 2.5. Assume that (2.1) holds, and let $f \in \overline{\mathfrak{D}}_{\infty}$ be given. If $\|f\|_{\infty}=0$, then $f$ is $\mu$-almost surely constant. If $\|f\|_{\infty}>0$, then $f \in L^{2}(\mu)$ and, in fact,

$$
\begin{array}{r}
\left\langle\exp \left[\frac{f}{\sqrt{2 P}\|f\|_{\infty}}\right]\right\rangle \leq K \exp \left[\frac{\langle f\rangle}{\sqrt{2 P}\|f\|_{\infty}}\right] \\
\text { where } K \equiv \prod_{1}^{\infty}\left(1-4^{-m}\right)^{-2^{m}}
\end{array}
$$

Proof. Because $\overline{\mathfrak{D}}_{\infty} \subseteq \overline{\mathfrak{D}}_{2}=D(\mathcal{E})$ and $\|f\|_{\infty} \geq\|f\|_{2}=\mathcal{E}(f, f)$ for $f \in$ $D(\mathcal{E})$, there is no question that $f \in L^{2}(\mu)$ and, from $(2.1)$, that $\|f\|_{\infty}=$ $0 \Longrightarrow f=\langle f\rangle \mu$-almost surely. Thus, assume that $0<\|f\|_{\infty} \leq 1$. By 
(1.15), $\left\|\psi_{n} \circ f\right\|_{\infty} \leq 1$ for all $n \in \mathbb{Z}^{+}$. Hence, we need only show that, for $f \in \mathfrak{D}_{\infty}$ satisfying $\|f\|_{\infty} \leq 1$ :

$$
\left\langle\exp \left[\frac{f}{\sqrt{2 P}}\right]\right\rangle \leq K \exp \left[\frac{\langle f\rangle}{\sqrt{2 P}}\right]
$$

To this end, set $\alpha=(2 P)^{-\frac{1}{2}}$ and

$$
u(t)=\left\langle e^{\alpha t f}\right\rangle, \quad t \in(0, \infty)
$$

Then, from (2.1) and (1.16):

$$
u(2 t) \leq P \mathcal{E}\left(e^{\alpha t f}, e^{\alpha t f}\right)+u(t)^{2} \leq t^{2} u(2 t)+u(t)^{2} .
$$

Thus, if $a_{n}=\log \left(u\left(2^{-n}\right)\right)$, then $a_{n-1} \leq-\log \left(1-4^{-n}\right)+2 a_{n}$, and so

$$
a_{0} \leq-\sum_{m=1}^{n} 2^{m} \log \left(1-4^{m}\right)+2^{n} a_{n}, \quad n \in \mathbb{Z}^{+}
$$

and, since $2^{n} a_{n} \longrightarrow(2 P)^{-\frac{1}{2}}\langle f\rangle$ as $n \rightarrow \infty$, the proof is complete.

Note that, because (cf. (1.14))

$$
\||f|\|_{p} \leq\|f\|_{p}, \quad \text { for all } p \in[2, \infty) \text { and } f \in \overline{\mathfrak{D}}_{p},
$$

the preceding shows that

$$
\left\langle\exp \left[\frac{|f|}{\sqrt{2 P}\|f\|_{\infty}}\right]\right\rangle \leq K \exp \left[\frac{\langle|f|\rangle}{\sqrt{2 P}\|f\|_{\infty}}\right] .
$$

Moreover (cf. Remark 1.17, when $\mathcal{E}$ is local, and therefore (1.18) holds, (2.18) can be replaced by

$$
\left\langle\exp \left[\frac{|f|}{\sqrt{P}\|f\|_{\infty}}\right]\right\rangle \leq K \exp \left[\frac{\langle|f|\rangle}{\sqrt{P}\|f\|_{\infty}}\right]
$$




\section{The logarithmic Sobolev inequality}

In this section we will examine what can be said in cases when one knows that Gross's logarithmic Sobolev inequality

$$
\left\langle f^{2} \log \frac{f^{2}}{\|f\|_{L^{2}(\mu)}^{2}}\right\rangle \leq G \mathcal{E}(f, f)+D\|f\|_{L^{2}(\mu)}^{2}, \quad f \in \mathcal{F},
$$

holds. Recall (cf. Corollary 6.1.17 and Theorem 6.1.22 in [4]) that

$$
\text { (3.1) with } D=0 \Longrightarrow(2.1) \text { with } 2 P \leq G,
$$

and, conversely,

$$
\text { (2.1) plus }(3.1) \Longrightarrow\left\langle f^{2} \log \frac{f^{2}}{\|f\|_{L^{2}(\mu)}^{2}}\right\rangle \leq \tilde{G} \mathcal{E}(f, f), \quad f \in \mathcal{F},
$$

where $\tilde{G} \leq G+(D+2) P$. Thus, when $D=0$, (3.1) is certainly stronger than (2.1), and, even when $D>0$, it gives additional information as soon as one knows $\|f\|_{L^{2}(\mu)}$. With this in mind, the following results should not be surprising.

Theorem 3.4. Assume (3.1), and let $p \in(2, \infty)$ be given. Then, $L^{2}(\mu) \cap$ $\overline{\mathfrak{D}}_{p} \subseteq L^{p}(\mu)$ and

$$
\begin{aligned}
\|f\|_{L^{p}(\mu)}^{2} & \leq e^{\frac{2 D}{p^{\prime}}}\left(\|f\|_{L^{2}(\mu)}^{2}+G \int_{2}^{p}\|f\|_{t}^{2} d t\right) \\
& \leq e^{\frac{2 D}{p^{\prime}}}\left(\|f\|_{L^{2}(\mu)}^{2}+G(p-2)\|f\|_{p}^{2}\right)
\end{aligned}
$$

for $f \in L^{2}(\mu) \cap \overline{\mathfrak{D}}_{p}$. In particular, when $D=0, \overline{\mathfrak{D}}_{p} \subseteq L^{p}(\mu)$ and (3.5) can be replaced by

$$
\|f\|_{L^{p}(\mu)}^{2} \leq\|f\|_{L^{2}(\mu)}^{2}+G \int_{2}^{p}\|f\|_{t}^{2} d t \leq\|f\|_{L^{2}(\mu)}^{2}+G(p-2)\|f\|_{p}^{2} .
$$

Proof. By (2.7), we can assume that $f>0$. In addition, it suffices to treat the case when $f \in \mathcal{F}$, since the general case then follows from consideration of $\psi_{n} \circ f$. Thus, let $f$ be a positive element of $\mathcal{F}$ with $\|f\|_{p}<\infty$ be given. Then,

$$
\frac{d}{d t}\left\langle f^{t}\right\rangle^{\frac{2}{t}}=\frac{2}{t^{2}}\left\langle f^{t}\right\rangle^{\frac{2}{t}-1}\left\langle f^{t} \log \frac{f^{t}}{\left\langle f^{t}\right\rangle}\right\rangle .
$$

Hence, by (3.1) and (1.16) and elementary manipulation,

$$
\frac{d}{d t}\left(e^{\frac{2 D}{t}}\left\langle f^{t}\right\rangle^{\frac{2}{t}}\right) \leq G e^{\frac{2 D}{t}}\|f\|_{t}^{2}
$$

for $t \geq 2$, and clearly the desired results all follow easily from this. 
Corollary 3.7. Again assume that (3.1) holds, but this time assume that $f \in \bigcap_{p \in[2, \infty)} \overline{\mathfrak{D}}_{p}$ and that there exist $C \in(0, \infty)$ and $\alpha \geq 0$ such that

$$
\|f\|_{p} \leq C p^{\alpha} \quad \text { for all } p \in[2, \infty) .
$$

Set $\beta=\frac{2}{2 \alpha+1}$. Then, for $\epsilon>0$ satisfying $2 \epsilon G^{\frac{1}{2}} e^{D}<1$ :

$$
\begin{aligned}
&\left\langle\exp \left[\frac{1}{e \beta}\left(\frac{\epsilon|f|}{C}\right)^{\beta}\right]\right\rangle \leq 2 \exp {\left[\frac{1}{e \beta}\left(\frac{2 \epsilon e^{D}\|f\|_{l} m 2}{C}\right)^{\beta}\right] } \\
&+S\left[1-\left(2 \epsilon G^{\frac{1}{2}} e^{D}\right)^{\beta}\right]^{-1}
\end{aligned}
$$

where $S \equiv \sup _{n \in \mathbb{Z}^{+}}(n !)^{-1}\left(\frac{n}{e}\right)^{n}$.

Proof. Again it suffices to treat the case in which $f \in \mathcal{F}$. By (3.5):

$$
\left\langle|f|^{n \beta}\right\rangle=\left(\|f\|_{L^{n \beta}(\mu)}^{2}\right)^{\frac{n \beta}{2}} \leq\left(2 e^{D}\right)^{n \beta}\left[\|f\|_{L^{2}(\mu)}^{n \beta}+\left(G^{\frac{1}{2}} e^{D} C\right)^{n \beta}(n \beta)^{n}\right]
$$

for $n \geq \frac{2}{\beta}$. Hence, since $\left\langle|f|^{n \beta}\right\rangle \leq\|f\|_{L^{2}(\mu)}^{n \beta}$ when $n \beta<2$, one gets (3.9) by using the preceding estimates in the power series expressions for the left hand side.

It is interesting that, in the case when $D=0$ and $f \in \overline{\mathfrak{D}}_{\infty}$, one can make a substantial improvement in (3.9). Namely, from (3.6), we know that if $u(t)=\left\langle e^{2 t f^{2}}\right\rangle$ for some $f \in \mathcal{F}$, then

$$
u^{\prime}(t)=\frac{u(t)^{1-t}}{t^{2}}\left\langle e^{2 t f^{2}} \log e^{2 t f^{2}}\right\rangle .
$$

Hence, if (3.1) holds with $D=0$ and $\|f\|_{\infty} \leq 1$, then

$$
u^{\prime}(t) \leq 8 G u(t)^{1-t}\left\langle f^{2} e^{2 t f^{2}}\right\rangle .
$$

But, clearly, another expression for $u^{\prime}$ is

$$
u^{\prime} t(t)=-\frac{1}{t} u(t) \log u(t)+\frac{2}{t} u(t)^{1-t}\left\langle f^{2} e^{2 t f^{2}}\right\rangle,
$$

and so we now see that $u^{\prime}(t) \leq 4 G t u^{\prime}(t)+4 G u(t) \log u(t)$. Finally, since $\lim _{t \searrow 0} u(t)=2\left\langle f^{2}\right\rangle$, we have proved the following theorem.

Theorem 3.10. Assume that (3.1) holds with $D=0$. Then $\overline{\mathfrak{D}}_{\infty} \subseteq$ $\bigcap_{p \in[2, \infty)} L^{p}(\mu)$. In fact, if $\|f\|_{\infty} \in(0, \infty)$ and $0<\epsilon<(2 G)^{-1}$, then

$$
\left\langle\exp \left[\frac{\epsilon f^{2}}{\|f\|_{\infty}}\right]\right\rangle \leq \exp \left[\frac{\epsilon\|f\|_{L^{2}(\mu)}}{(1-2 \epsilon G)\|f\|_{\infty}}\right] \text {. }
$$

Remark 3.12. When $\mathcal{E}$ is local and (cf. Remark 1.1.7) therefore (1.18) holds, $2 G$ can be replaced by $G$ in each of the estimates (3.5), (3.9), and (3.11). 


\section{An example}

Applications of the type of results in Sections 2 and 3 have already been given in the setting of Riemannian geometry (cf. [3]) and abstract Wiener spaces by several authors (cf. [7], [5], and [1]). In both of these settings, $\mathcal{E}(f, f)=\|\nabla f\|_{L^{2}(\mu)}^{2}$, where $\nabla$ is a local gradient operation. To show how such results might be applicable in a non-local setting, take $E=\{-1,1\}^{\mathbb{Z}^{d}}$ and let

$$
|\nabla f(\omega)|=\left(\sum_{\mathbf{k} \in \mathbb{Z}^{d}}\left|\partial_{k} f(\omega)\right|^{2}\right)^{\frac{1}{2}}
$$

where $\partial_{k} f(\omega) \equiv f\left(\omega^{\mathbf{k}}\right)-f(\omega)$ and $\omega^{k}$ is the element of $E$ obtained from $\omega$ by replacing the kth coordinate with $-\omega_{\mathbf{k}}$. Next, let $\mu$ be a Gibbs state on $E$ corresponding to a finite range potential $\mathcal{J}$. As was shown in $[2], \mu$ will satisfy the Poincaré inequality

$$
\operatorname{var}(f) \leq P\|\nabla f\|_{L^{2}(\mu)}^{2}, \quad f \in L^{2}(\mu)
$$

for some $P$ whenever $\mathcal{J}$ satisfies the uniqueness condition of Dobrushin and Shlosman. Moreover, it was shown in [6] that the mixing condition of Dobrushin and Shlosman is sufficient to guarantee the logarithmic Sobolev inequality

$$
\left\langle f^{2} \log \frac{f^{2}}{\|f\|_{L^{2}(\mu)}^{2}}\right\rangle \leq G\|\nabla f\|_{L^{2}(\mu)}^{2}, \quad f \in L^{2}(\mu)
$$

for some $G$.

Now assume that $\mathcal{J}$ is shift-invariant and satisfies the Dobrushin-Shlosman uniqueness condition. Then $\mu$ is shift-invariant and satisfies (4.1). Next, let $\psi: E \longrightarrow \mathbb{R}$ be a continuous function for which $\langle\psi\rangle=0$ and

$$
B \equiv \sum_{\mathbf{k} \in \mathbb{Z}^{d}}\left\|\partial_{\mathbf{k}} \psi\right\|_{L^{\infty}(\mu)}<\infty
$$

Finally, suppose that $\left\{a_{\mathbf{k}}: \mathbf{k} \in \mathbb{Z}^{d}\right\} \subseteq \mathbb{R}$ is square summable with $A=$ $\left(\sum_{\mathbf{k} \in \mathbb{Z}^{d}} a_{\mathbf{k}}^{2}\right)^{\frac{1}{2}}$, set $f_{0}=0$, and, for $n \in \mathbb{Z}^{+}$,

$$
f_{n}=\sum_{|\mathbf{j}|<n} a_{\mathbf{j}} \psi \circ S^{\mathbf{j}}
$$


where $S^{\mathbf{j}}$ is the natural shift on $E$ determined by $S^{\mathbf{j}}(\omega)_{\mathbf{k}}=\omega_{\mathbf{j}+\mathbf{k}}$. Clearly,

$$
\left|\nabla\left(f_{n}-f_{m}\right)\right|=\left(\sum_{\ell \in \mathbb{Z}^{d}}\left(\sum_{m \leq|\mathbf{j}|<n} a_{\mathbf{j}}\left(\partial_{\mathbf{j}-\mathbf{k}} \psi\right) \circ S^{\mathbf{j}}\right)^{2}\right)^{\frac{1}{2}},
$$

and so, by Young's inequality,

$$
\left\|f_{n}-f_{m}\right\|_{\infty}=\left\|\nabla\left(f_{n}-f_{m}\right)\right\|_{L^{\infty}(\mu)} \leq B\left(\sum_{|\mathbf{j}| \geq m} a_{\mathbf{j}}^{2}\right)^{\frac{1}{2}} .
$$

In particular, by (4.1), this proves that $f_{n}$ converges in $L^{2}(\mu)$ to an $f \in \overline{\mathfrak{D}}_{\infty}$ for which $\|f\|_{L^{2}(\mu)} \leq \sqrt{P} A$ and $\|f\|_{\infty} \leq A B$. In fact, by (2.6), we know that

$$
\left\langle\exp \left[\frac{f}{\sqrt{2 P} A B}\right]\right\rangle \leq K
$$

Moreover, if, in addition, (4.2) holds, then

$$
\left\langle\exp \left[\frac{\epsilon f^{2}}{A B}\right]\right\rangle \leq \exp \left[\frac{\epsilon P A}{(1-2 \epsilon G) B}\right] .
$$

Notice the similarity between these estimates and those which are familiar in the non-interacting case (i.e. when $\mu=\lambda^{\mathbb{Z}^{d}}$ for some $\lambda$ on $\{-1,1\}$ ). This is what we had in mind in $\S 0$ when we said that, a Poincaré inequality and, even more so, a logarithmic Sobolev inequality is an expression of strong mixing properties of the measure involved.

\section{References}

1. S. Aida, T. Masuda, and I. Sigekawa, Logarithmic Sobolev inequalities and exponential integrability.

2. M. Aizenman and R. Holley, Rapid convergence to equilibrium of stochastic Ising Models in the Dobrushin Shlosman regime, Percolation Theory and Ergodic Theory of Infinite Particle Systems (H. Kesten, ed.), IMS Volumes in Math. and Appl., Springer-Verlag, New York, 1987, pp. 1-11.

3. A. Brooks, A relation between growth and the spectrum of the Laplacian, Math. Zeit. (1981), no. 178, 501-508.

4. R. L. Dobrushin and S. B. Shlosman, Constructive criterion for the uniqueness of Gibbs field, Statistical Physics and Dynamical Systems, Rigorous Results (Fritz, Jaffe, and Szasz, eds.), Birkhäuser, 1985, pp. 347-370.

5. S. Kusuoka, Analysis on Wiener space, J. Fnal. Anal. (1991), no. 98, 122-168.

6. D. Stroock and B. Zegarlinski, The logarithmic Sobolev inequality for discrete spin systems on a lattice, Comm. Math. Phys. (1992), no. 149, 175-193. 
7. A. S. Üstünel, Intégrabilité exponential de fonctionnelles de Wiener, C.R. Acad. Sci. Paris, Serie 1315 (1992), no. 9, 997-1000.

Mathematical Institute, Tôhoku University, Sendai 980 , Japan

E-mail address: aida@math.mit.edu

M IT, 2-272, CAMBRIDGE, M A, 02139 , U S A

E-mail address: dws@math.mit.edu 